\title{
Radiation therapy at compact Compton sources
}

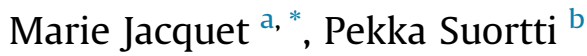 \\ a Laboratoire de l'Accélérateur Linéaire, Univ. Paris-Sud et IN2P3/CNRS, Orsay, France \\ ${ }^{\mathrm{b}}$ Department of Physics, University of Helsinki, POB 64, FIN-00014 Helsinki, Finland
}

\section{A R T I C L E I N F O}

\section{Article history:}

Received 20 December 2014

Received in revised form

27 January 2015

Accepted 16 February 2015

Available online 6 March 2015

\section{Keywords:}

X-rays

Compton source

Radiation therapy

\begin{abstract}
A B S T R A C T
The principle of the compact Compton source is presented briefly. In collision with an ultrarelativistic electron bunch a laser pulse is back-scattered as hard X-rays. The radiation cone has an opening of a few mrad, and the energy bandwidth is a few percent. The electrons that have an energy of the order of a few tens of $\mathrm{MeV}$ either circulate in storage ring, or are injected to a linac at a frequency of $10-100 \mathrm{MHz}$. At the interaction point the electron bunch collides with the laser pulse that has been amplified in a Fabry-Perot resonator. There are several machines in design or construction phase, and projected fluxes are $10^{12}$ to $10^{14}$ photons/s. The flux available at $80 \mathrm{keV}$ from the ThomX machine is compared with that used in the Stereotactic Synchrotron Radiation Therapy clinical trials. It is concluded that ThomX has the potential of serving as the radiation source in future radiation therapy programs, and that Thom X can be integrated in hospital environment.

(C) 2015 Published by Elsevier Ltd on behalf of Associazione Italiana di Fisica Medica. This is an open access article under the CC BY-NC-ND license (http://creativecommons.org/licenses/by-nc-nd/4.0/).
\end{abstract}

\section{Introduction}

The use of synchrotron radiation (SR) has opened new avenues in biomedical research [1,2]. 3-dimensional structures of macroscopic objects can be imaged with a resolution of a few microns and elemental distributions in cells can be mapped with spatial resolution better than $100 \mathrm{~nm}$. These are made possible by the high intensity and natural collimation, energy tunability, and partial transverse coherence of SR, which are utilized in phase contrast imaging and in X-ray micro-spectroscopy.

The limited availability of SR has curtailed the applications mostly to basic research, and to methods development with the distant goal of clinical use. Only a few programs have reached the stage of human studies, the first being coronary angiography with intravenous injection of the Iodine contrast agent. For these studies a new method, K-edge subtraction (KES) imaging was developed, and has been applied in studies of circulation and ventilation in animal models. The radiation dose in KES imaging is within clinically acceptable limits, and there are many potential applications in functional imaging of human organs [3]. Phase contrast imaging in the propagation mode has been used in mammography, demonstrating enhanced contrast resolution [4]. Clinical trials for radiation therapy of brain tumors is underway at the European

\footnotetext{
* Corresponding author.

E-mail address: mjacquet@lal.in2p3.fr (M. Jacquet).
}

Synchrotron Radiation Facility (ESRF) using the Stereotactic Synchrotron Radiation Therapy (SSRT) method [5].

There have been many projects for transfer of phase contrast imaging methods to clinical environment without the use of large scale synchrotrons see Ref. [2]. In most cases, rotating anode or micro-focus X-ray tubes are used as radiation sources, and new concepts have been introduced to overcome the anode cooling problems. The anode may be replaced by a wire or thin stream of liquid metal, and potentially these sources are at least an order of magnitude brighter than conventional X-ray tubes. However, even with these improvements the exposure times would be prohibitively long for most modalities of human imaging.

Probably the only way to break out of the confinement of scarce synchrotron radiation at large facilities on one hand, and the insufficient X-ray flux from tube sources on the other, is the introduction of compact SR sources for dedicated medical imaging and/or therapy facilities. Most concepts of compact sources are based on the use of very high-field magnets in small storage rings, but still the X-ray energies remain too low for most imaging applications, and totally exclude radiation therapy [6]. A different way of producing X-rays from a high-energy electron beam is to collide the beam with a laser beam, which is scattered back in a process that is usually called inverse Compton scattering [7]. Originally, the method was introduced in high-energy particle physics, but more than a decade ago it was demonstrated that a linac-laser combination produces short, intense X-ray pulses of sufficient intensity for medical imaging [8]. In recent years, many projects have been 
initiated, and the first and the only compact inverse-Compton source on market is the Lynceantech machine, which delivers Xrays in the range $15-35 \mathrm{keV}$ [9]. It is expected that these sources will become standard equipment for clinical imaging in large hospitals.

\section{Inverse Compton scattering and compact Compton sources}

The inverse Compton scattering process is shown schematically in Fig. 1(a). In the collision, the energy of the electron is transmitted to the photon. The kinematics of the process can be described as collision of two particles, or using the synchrotron radiation analogy, where the electron travels through the "micro-undulator" of the electromagnetic field of the photon. The energy of the scattered photon in the laboratory frame is [10]:

$\mathrm{E}_{\mathrm{X}}=2 \gamma^{2} \mathrm{E}_{\mathrm{L}}\left(1+\cos \theta_{\mathrm{c}}\right) /\left(1+\gamma^{2} \theta^{2}\right)$,

where $\gamma$ is the electron energy in the units of its rest energy, $\gamma=E_{e} / m c^{2}, E_{L}$ the energy of the incoming photon, $\theta_{c}$ and $\theta$ respectively the incident angle and the scattering angle with respect to the electron trajectory. In derivation of (1), it is assumed that $\gamma \gg 1$ and $E_{L} \ll m c^{2}$. Expression (1) shows that there is an univocal relation between the energy and the emission angle of Compton photons, the hardest radiation being concentrated onaxis where $\theta=0$. So in head-on collision (i.e. when $\theta_{c}=0$ ), the maximum energy of the scattered photons is $\mathrm{E}_{\mathrm{X}}^{\max }=4 \gamma^{2} \mathrm{E}_{\mathrm{L}}$. For instance, $0.1 \mathrm{~nm}(12.4 \mathrm{keV}) \mathrm{X}$-rays are produced in collision of a laser pulse of $1.0 \mu \mathrm{m}$ wavelength $\left(\mathrm{E}_{\mathrm{L}}=1.24 \mathrm{eV}\right)$ with a $25 \mathrm{MeV}$ electron bunch. The normalized spectral cross section $\sigma_{\omega}(\omega)$, where $\omega=E_{X} / E_{X}^{\max }$, is represented on Fig. 1(b) and can be written as [11]:

$\sigma(\omega)=3 / 2\left(1-2 \omega+2 \omega^{2}\right)$.

For electron/laser collisions in the $\mathrm{x}-\mathrm{z}$ plane, the number of $\mathrm{X}$-rays emitted per second is:

$F_{\text {tot }}=\frac{\sigma_{\mathrm{T}} \mathrm{N}_{\mathrm{e}} \mathrm{N}_{\mathrm{L}} \mathrm{f}_{\text {rep }} \cos \left(\theta_{\mathrm{c}} / 2\right)}{2 \pi \sqrt{\sigma_{\mathrm{ye}}^{2}+\sigma_{\mathrm{yL}}^{2}} \sqrt{\sigma_{\mathrm{xe}}^{2}+\sigma_{\mathrm{xL}}^{2}+\sin ^{2}\left(\theta_{\mathrm{c}} / 2\right)\left(\sigma_{\mathrm{ze}}^{2}+\sigma_{\mathrm{zL}}^{2}\right)}}$

where $\sigma_{\mathrm{T}}$ is the Thomson cross-section, $\mathrm{N}_{\mathrm{e}}$ the number of electrons per bunch, $\mathrm{N}_{\mathrm{L}}$ that of photons per laser pulse, and $\mathrm{f}_{\mathrm{rep}}$ the repetition rate of collisions. The transverse and longitudinal sizes of the (Gaussian) electron bunches and laser pulses at the interaction point are given by $\sigma_{x e, y e}=\sigma_{e}, \sigma_{x L, y L}=\sigma_{L}, \sigma_{z e}$ and $\sigma_{z L}$ respectively. The charge of an electron bunch may be $0.1-1.0 \mathrm{nC}$, so $N_{e}$ is about $10^{9}$, and the repetition rate is between $10^{7} 1 / \mathrm{s}$ and $10^{8} 1 / \mathrm{s}$. To give a concrete example with feasible values of $\mathrm{f}_{\mathrm{rep}}=20 \mathrm{MHz}, 1 \mathrm{nC}$ electron bunches, and $10 \mathrm{~mJ}$ laser pulses of $1 \mu \mathrm{m}$ wavelength, $F \sim 10^{13} \mathrm{ph} / \mathrm{s}$ is expected when $\sigma_{e}=\sigma_{L}=40 \mu \mathrm{m}$.
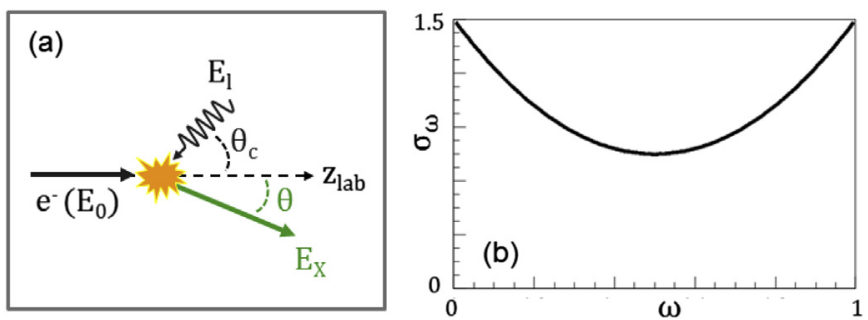

Figure 1. Inverse Compton scattering process (a) and normalized Compton cross section as a function of $\omega=E_{\mathrm{X}} / \mathrm{E}_{\mathrm{X}}^{\max }(\mathrm{b})$.
There are basically two machine designs: the storage ring scheme and the linac scheme, illustrated in Fig. 2. Each one has advantages and technical difficulties. In the storage ring scheme each stored electron bunch interacts many times with a laser pulse. The bunch is blurred by intra-beam scattering and Compton scattering, so that it is ejected and replaced by a new bunch. The repetition frequency is determined by the ring circumference, for instance $\mathrm{f}_{\mathrm{rep}}$ is $20 \mathrm{MHz}$ when the circumference of $15 \mathrm{~m}$. In the linac scheme each electron bunch interacts only once, and $f_{\text {rep }}$ is determined by the electron gun, which is necessarily a superconducting device. The advantage of the linac scheme is the small transverse size of the electron bunch, so that the transverse coherence of the $\mathrm{X}$-ray beam is high. However, continuous cryogenic operation of the electron gun with high repetition rate entails many technical and operational challenges, particularly in clinical environment. In both schemes, intense laser pulses at frequency $f_{\text {rep }}$ are created by the amplification of pulses from a low average power pulsed laser which are then sent in a high gain optical resonator for a second amplification stage. For instance, a $10 \mathrm{~mJ}$ laser pulse circulating at $20 \mathrm{MHz}$ frequency corresponds to $0.2 \mathrm{MW}$ stored in the optical cavity.

The properties of the X-ray beam depend on the energy spreads of the electron and laser beams, $\mathrm{d} \gamma$ and $\mathrm{dE}_{\mathrm{L}}$, respectively, and on the divergences of the beams. The relative bandwidth of on-axis photons due to energy spreads of the beams is approximately $\mathrm{dE}_{\mathrm{L}}$ / $E_{L}+2 d \gamma / \gamma$, which is typically of the order of $10^{-3}$. More important are the effects of beam divergences, and the dominant factor is the electron beam divergence $\sigma_{\text {xe,ye }}^{\prime}=\sigma_{\mathrm{e}}^{\prime}$, yielding $\mathrm{d} \mathrm{E}_{\mathrm{X}} / \mathrm{E}_{\mathrm{X}}=2\left(\gamma \sigma_{\mathrm{e}}^{\prime}\right)^{2}$. With typical values of $\gamma=100$ (50 MeV electron beam) and $\sigma_{\mathrm{e}}^{\prime}=1 \mathrm{mrad}$, the relative energy spread of the X-ray beam is $2 \%$.

The brilliance $\mathrm{B}$ of the source is given as the number of X-ray photons per second, per $\mathrm{mm}^{2}$ of source, in solid angle of $1 \mathrm{mrad}^{2}$, and in $0.1 \%$ spectral bandwidth. Considering only the dominant term due to the electron beam divergence, on-axis $\mathrm{B}$ is proportional to $\mathrm{F} \gamma^{2} / \varepsilon_{\mathrm{N}}$, where $\varepsilon_{\mathrm{N}}=\gamma \sigma_{\mathrm{e}} \sigma_{\mathrm{e}}^{\prime}$ is the normalized (tranverse) emittance of the electron beam [10]. In the same way as for the degree of transverse coherence, the electron beam emittance is the crucial factor for the X-ray beam brilliance.
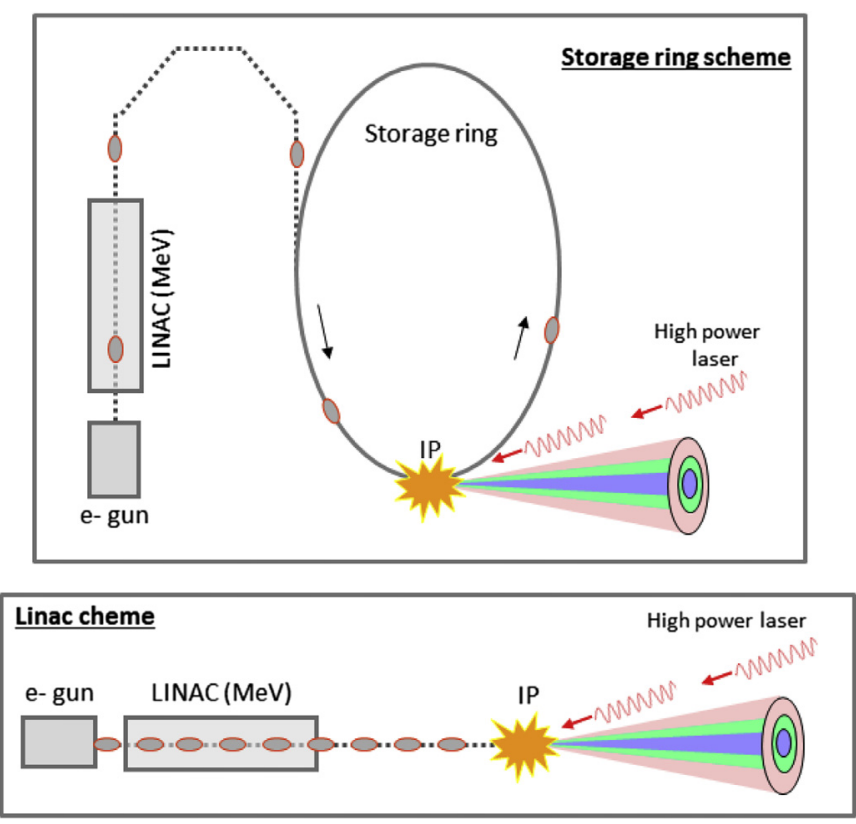

Figure 2. Basic schemes of high-flux compact Compton sources. IP is the interaction point of the laser pulse with the electron bunch. 


\section{Compact Compton source projects}

For biomedical applications, the compact source intensity (ph/s/ $\operatorname{mrad}^{2}$ ) should be comparable to the intensity of monochromated bending magnet radiation of synchrotron sources, i.e. $10^{11}-10^{12} \mathrm{ph} /$ $\mathrm{s} / \mathrm{mrad}^{2}$. In addition to the existing Lynceantech machine, several sources of higher intensity and higher X-ray energies are being designed [10]. Some characteristics and orders of magnitude of these are collected in Table 1.

The superconducting linac-based machines provide the highest brilliance but still face many technical challenges and will require extensive radiation shielding. For many medical imaging methods and for radiation therapy in particular the brilliance is not critical, but more important is the flux available in a given solid angle. Therefore, one of the storage-ring machines in advanced design/ construction phase is taken as a concrete example. The essential parameters of the ThomX machine [12] are given in Table 2.

Selecting a part of a Compton beam with a diaphragm of half angular aperture $\theta_{\mathrm{mr}}$ of a few mrad, the flux of photons $\mathrm{F}_{\theta_{\mathrm{mr}}}$ contained in this radiation cone and the energy bandwidth can be written with a good approximation as:

$\mathrm{F}_{\theta_{\mathrm{mr}}}=\mathrm{F}_{\mathrm{tot}}\left(\gamma^{2} \theta_{\mathrm{mr}}^{2}\right) \sigma\left(\omega_{\mathrm{mr}}\right)$,

$\mathrm{dE}_{\mathrm{X}} / \mathrm{E}_{\mathrm{X}} \sim \sqrt{\left(\gamma^{2} \theta_{\mathrm{mr}}^{2}\right)^{2}+\left(2 \gamma^{2} \sigma_{\mathrm{e}}^{\prime 2}\right)^{2}+(2 \mathrm{~d} \gamma)^{2}}$

where $\omega_{\mathrm{mr}}=1-\left(\gamma \theta_{\mathrm{mr}}\right)^{2}$. The first term in (5) is due to the univocal energy/angle relation (1), the two last terms are due to the divergence and the energy spread of the electron beam respectively. Results of calculation using parameters of Table 2 with a value of $67 \mathrm{MeV}$ for the electron beam energy are shown in Fig. 3. The total flux and the flux in different opening angles of radiation cone are shown as a function of $\sigma_{\mathrm{e}}$ and $\sigma_{\mathrm{e}}^{\prime}$ (top) and as a function of the X-ray energy bandwidth (bottom). According to (1), (3), (4) and (5), fluxes decrease as $\sigma_{\mathrm{e}}$ increases, the energy bandwidth increases with the opening angle $\theta_{\mathrm{mr}}$ and with the divergence $\sigma_{\mathrm{e}}^{\prime}$ of the electron beam. These plots are used for assessment of the suitability of ThomX for radiation therapy.

\section{Stereotactic Synchrotron Radiation Therapy (SSRT)}

The only synchrotron radiation therapy program that has reached the stage of human studies is the Stereotactic Synchrotron Radiation Therapy at the ESRF. The technical parameters and the protocol have been optimized for treatment of brain tumors, which have been "loaded" by an Iodine compound for radiation dose enhancement. As a compromise between beam penetration through the skull and excitement of Iodine fluorescence and secondary electrons, $80 \mathrm{keV}$ incident radiation is used. The beam from

\section{Table 1}

X-ray compact Compton source projects aiming to produce a flux larger than $10^{12} \mathrm{ph} / \mathrm{s}$ [10]. SR and Linac mean Storage Ring and Linac schemes, respectively, and (sc) indicates the projects using superconducting technologies. $\sigma_{\mathrm{S}}$ is the transverse source size.

\begin{tabular}{llllll}
\hline Project & Type & $\mathrm{E}_{\mathrm{X}}(\mathrm{keV})$ & $\mathrm{ph} / \mathrm{s}$ & $\mathrm{ph} / \mathrm{s} / \mathrm{mrad}^{2}$ & $\sigma_{\mathrm{S}}(\mu \mathrm{m})$ \\
\hline TTX & SR & $20-80$ & $10^{12}$ & $6.10^{9}-2.10^{10}$ & 50 \\
LEXG & SR (sc) & 33 & $10^{13}$ & $10^{11}$ & 20 \\
NESTOR & SR & $30-500$ & $10^{13}$ & $10^{11}-10^{12}$ & 70 \\
ThomX & SR & $20-90$ & $10^{13}$ & $6.10^{10}-3.10^{11}$ & 70 \\
KEK QB & Linac (sc) & 35 & $10^{13}$ & $10^{11}$ & 10 \\
KEK ERL & Linac (sc) & 67 & $10^{13}$ & $2.10^{11}$ & 30 \\
MIT & Linac (sc) & $3-30$ & $10^{14}$ & $10^{11}-10^{12}$ & 2 \\
\hline
\end{tabular}

Table 2

Nominal collision parameters of the ThomX project. $\varepsilon_{\mathrm{N}}$ and $\mathrm{d} \gamma$ are the mean of the emittance and energy spread on one fill of $20 \mathrm{~ms}$.

\begin{tabular}{lll}
\hline ThomX nominal parameters & & \\
\hline Interaction angle $\left(\theta_{\mathrm{c}}\right)$ & 2 & Degree \\
Repetition rate $\left(\mathrm{f}_{\mathrm{rep}}\right)$ & 17.8 & $\mathrm{MHz}$ \\
Injection frequency & 50 & $\mathrm{~Hz}$ \\
Electron energy & $50-70$ & $\mathrm{MeV}$ \\
Electron bunch charge & 1 & $\mathrm{nC}$ \\
Electron normalized emittance $\left(\varepsilon_{\mathrm{N}}\right)$ & 10 & $\mathrm{~mm} . \mathrm{mrad}$ \\
Electron energy spread $(\mathrm{d} \gamma)$ & 0.35 & $\%$ \\
Laser wavelength & 1.03 & $\mu \mathrm{m}$ \\
Laser pulse energy & 30 & $\mathrm{~mJ}$ \\
Laser pulse transverse size $\left(\sigma_{\mathrm{L}}\right)$ & 40 & $\mu \mathrm{m}$ \\
\hline
\end{tabular}
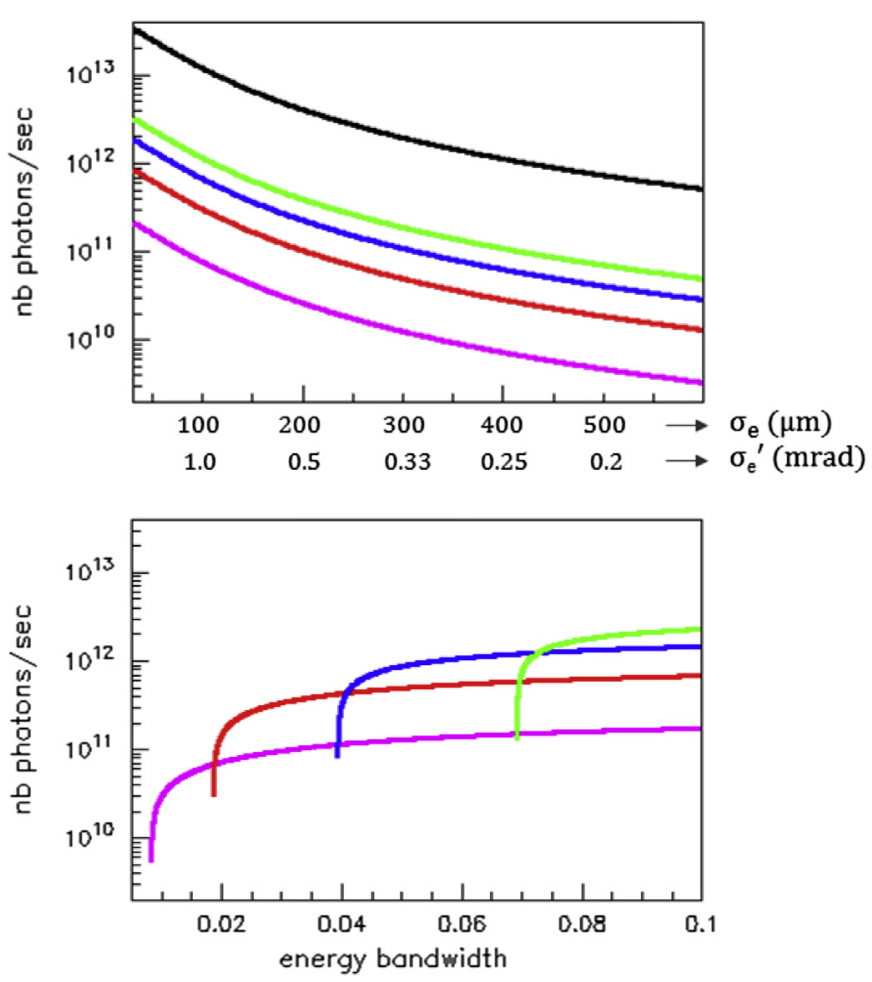

Figure 3. X-ray flux to different opening angles of the radiation cone as a function of $\sigma_{\mathrm{e}}$ and $\sigma_{\mathrm{e}}^{\prime}$ (top) and as a function of the X-ray energy bandwidth (bottom). The electron energy is $67 \mathrm{MeV}$, and the average normalized emittance during a $20 \mathrm{~ms}$ fill of the storage ring is $\varepsilon_{\mathrm{N}}=10 \mathrm{~mm} . \mathrm{mrad}$. Total flux (black), fluxes in $0.5 \mathrm{mrad}$ (purple), $1 \mathrm{mrad}$ (red), $1.5 \mathrm{mrad}$ (blue) and $2 \mathrm{mrad}$ (green). (For interpretation of the references to color in this figure caption, the reader is referred to the web version of this article.)

a multipole wiggler is filtered by carbon, aluminum, and copper foils to remove soft radiation and reduce the heat load on the optical elements of the beamline. About $0.1 \%(80 \mathrm{eV})$ energy band is separated by a bent-crystal, double-Laue monochromator [13]. Typically, the beam height is $2 \mathrm{~mm}$, and the maximum width is $150 \mathrm{~mm}$. The measured skin entry dose rate is $0.5 \mathrm{~Gy} / \mathrm{s}$, when the storage ring current is $200 \mathrm{~mA}$. This corresponds to $1.6 \times 10^{10} \mathrm{ph} / \mathrm{s} /$ $\mathrm{mm}^{2}$ of $80 \mathrm{keV}$ photons.

In the treatment the dose is averaged by moving the patient vertically. The radiation treatment set-up is shown in Fig. 4. The exposed area is defined by a hole in mask, which corresponds to the tumor area seen from that direction. The tumor is imaged at the hospital with a CT scanner, and the location and tumor dimensions are verified by CT imaging at the beamline. If the height of the beam is $2.0 \mathrm{~mm}$, and the vertical opening of the mask $30 \mathrm{~mm}$, the average dose rate is $35 \mathrm{mGy} / \mathrm{s}$. This corresponds to 


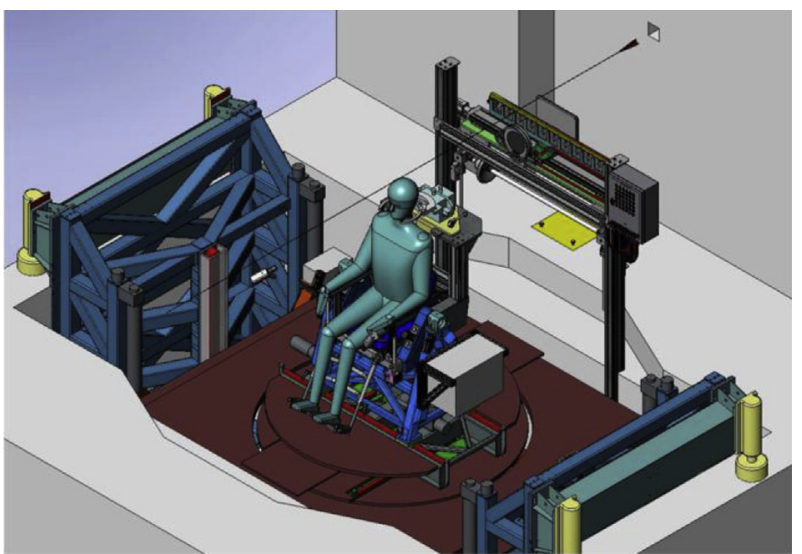

Figure 4. The SSRT clinical trials set-up at ID17 of the ESRF. The incident beam (top, right) passes through an aperture that corresponds to the tumor cross-section.

about $1.1 \times 10^{9} \mathrm{ph} / \mathrm{s} / \mathrm{mm}^{2}$. At the typical tumor location the absorbed dose is reduced to about $0.5 \mathrm{~Gy} / \mathrm{min}$. The total dose at the tumor depends on the number of irradiation ports (maximum 10 ), and the speed of the vertical scan. For safety reasons and for regulation of the dose, many fast scans are performed instead of one slow scan. The absorbed dose at the tumor is proportional to the total irradiation time, for instance $10 \mathrm{~Gy}$ is delivered in $20 \mathrm{~min}$.

There is a wide margin for increase of the dose. ID17 has two wigglers of approximately equal radiation powers, and the height of the monochromatic beam can be doubled. In addition, the dose enhancement at the tumor may be substantially increased by the use of a more concentrated Iodine solution in the tumor circulation. The clinical trials are in Phase II at the moment, with 7 patients treated so far, and in the forthcoming trials therapeutic doses (several tens of Gy) will be used.

\section{SSRT with ThomX}

The above analysis of the SSRT clinical trials gives guidelines and benchmarks for the potential use of ThomX in radiation therapy. In the following calculations the value of $67 \mathrm{MeV}$ for the electron beam energy is assumed. According to (1), this leads to $80.5 \mathrm{keV}$ onaxis X-rays.
It is inferred from Fig. 3 that ThomX could provide an X-ray flux from $1.9 \times 10^{12}$ to $4.1 \times 10^{10}$ in a cone of $1.5 \mathrm{mrad}$ half opening angle depending on the values of the transverse size and the divergence of the electron beam (blue curve on the top figure). Figure 3 shows also the bandwidth in that $1.5 \mathrm{mrad}$ cone (blue curve on the bottom figure): for instance $0.88 \times 10^{12} \mathrm{ph} / \mathrm{s}$ in $5 \%$ bandwidth should be available $\left(\sigma_{\mathrm{e}}=80 \mu \mathrm{m}\right.$ and $\sigma_{\mathrm{e}}^{\prime}=0.94 \mathrm{mrad}$ in this case) and $1.5 \times 10^{12} \mathrm{ph} / \mathrm{s}$ in $10 \%$ bandwidth (if $\sigma_{\mathrm{e}}=47 \mu \mathrm{m}$ and $\sigma_{\mathrm{e}}^{\prime}=1.6 \mathrm{mrad}$ ). Through a mask opening of $30 \mathrm{~mm}$ diameter placed at $10 \mathrm{~m}$ from the ThomX interaction point, the corresponding intensities are about $1.25 \times 10^{9} \mathrm{ph} / \mathrm{s} / \mathrm{mm}^{2}$ and $2.1 \times 10^{9} \mathrm{ph} / \mathrm{s} / \mathrm{mm}^{2}$ respectively. These values are very close to the average intensity in the present SSRT clinical trials at the ESRF. If required, the absorbed dose at the tumor may be increased by a more concentrated Iodine solution and by decreasing to distance of the patient from the X-ray source; $10 \mathrm{~m}$ used in the above calculations is actually a rather conservative estimate. A schematic layout of the ThomX machine for radiation therapy is shown in Fig. 5.

The design parameters of ThomX are based on existing and proven technical solutions. The storage ring scheme is easily adapted to hospital environment. The fill frequency is only $50 \mathrm{~Hz}$, so the energy stored in the electron beam is a few watts only, and little radiation shielding is required. In addition, the machine could be routinely operated by non-expert staff provided that simple control tools are developed and integrated. The most critical component is probably the Fabry-Perot resonator, which must be well isolated from all sources of vibrations. On the other hand, superconducting machines may be more difficult to integrate and operate because of the requirement of an extensive radiation shielding and the complications related to cryogenic systems. The electron gun operates at $10-100 \mathrm{MHz}$ injection frequency leading to a stored energy of several tens of kilowatts.

\section{Conclusions}

The comparison of the X-ray intensity available from ThomX with the average intensity in the ongoing clinical trials of SSRT at the ESRF demonstrates the feasibility of radiation therapy with a compact Compton source (CCS). A CCS based on the storage ring scheme is compatible with clinical environment, and the CCS can be used also for medical imaging modalities that have been developed at synchrotron radiation facilities.

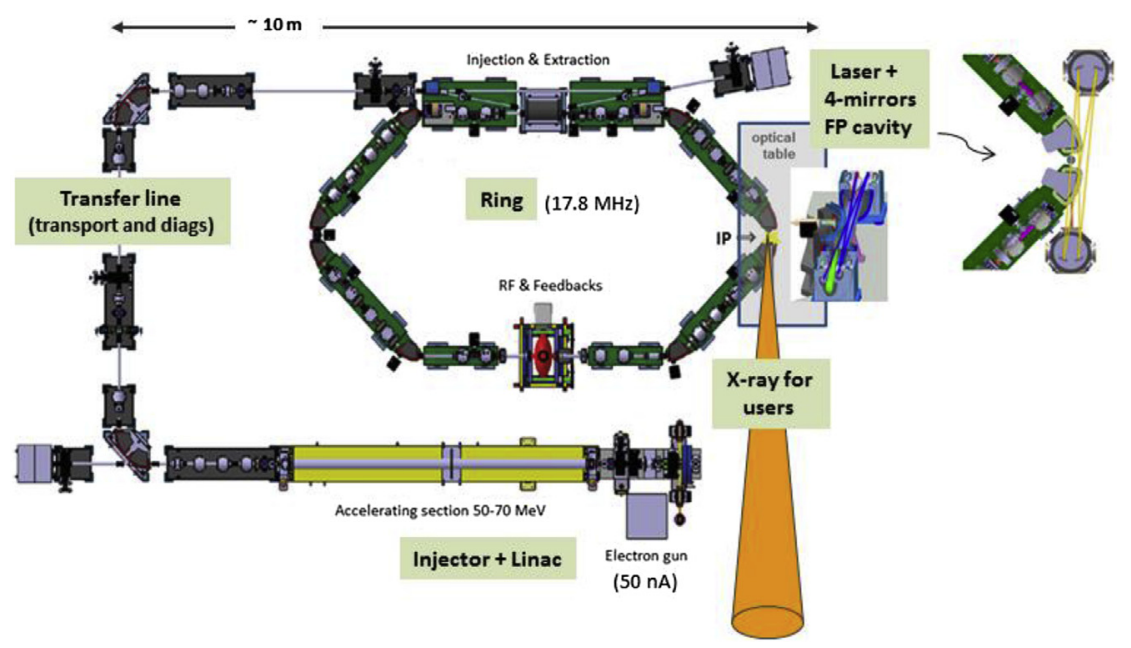

Figure 5. The layout of ThomX. 


\section{Acknowledgments}

The authors thank Hélène Elleaume and Michel Renier for making available the details of the SSRT clinical trials at the ESRF and thank the action COST-TD1205 allowing constructive work and many discussions.

\section{References}

[1] Suortti P, Thomlinson W. Medical applications of synchrotron radiation. Phys Med Biol 2003;48:R1-35.

[2] Bravin A, Coan P, Suortti P. X-ray phase-contrast imaging: from pre-clinical applications towards clinics. Phys Med Biol 2013;58:R1-35.

[3] Bertrand B, Estève F, Elleaume H, Nemoz C, Fiedler S, Bravin A, et al. Comparison of synchrotron radiation angiography with conventional angiography for the diagnosis of in-stent restenosis after percutaneous transluminal coronary angioplasty. Eur Heart J 2005;26:1284-91.

[4] Castelli E, Tonutti M, Arfelli F, Longo R, Quaia E, Rigon L, et al. Mammography with synchrotron radiation: first clinical experience with phase-detection technique. Radiology 2011;259(3):684-94.
[5] Prezado Y, Fois G, Edouard M, Nemoz C, Renier M, Requardt H, et al. Biologica equivalent dose studies for dose escalation in the stereotactic synchrotron radiation therapy clinical trials. Med Phys 2009;36(3):725-33.

[6] Weihreter E. Compact synchrotron light sources, series on synchrotron radiation techniques and applications, Vol. 3. World Scientific; 1996.

[7] Federici L. The LADON photon beam with the ESRF $5 \mathrm{GeV}$ machine. Nuovo Cimento Lettr 1980;27:339-47.

[8] Carroll FE. Tunable monochromatic X-rays: a new paradigm in medicine. Am Roentgenol 2002;179(3):583-90.

[9] Achterhold K, Bech M, Schleede S, Potdevin G, Ruth R, Loewen R, et al Monochromatic computed tomography with a compact laser-driven X-ray source. Sci Rep 2013;3:1313.

[10] Jacquet M. High intensity compact compton X-ray sources: challenges and potential of applications. Nucl Instrum Methods B 2014;331:1-5.

[11] Telnov V. Laser cooling of electron beams at linear colliders. Nucl Instrum Methods A 2000;455:80-9.

[12] Variola A, Haissinski J, Loulergue A, Zomer F, editors. ThomX Technical Design Report; 2014. p. 164. <in2p3-00971281>.

[13] Suortti P, Fiedler S, Bravin A, Brochard T, Mattenet M, Renier M, et al. Fixedexit monochromator for computed tomography with synchrotron radiation at energies 18 to keV. J Synchrotron Rad 2000;7:340-7. 